\title{
Gravity anomalies and lithospheric flexure around the Longmen Shan deduced from combinations of in situ observations and EGM2008 data
}

\author{
Yawen She, Guangyu Fu*, Zhuohua Wang, Tai Liu, Changyi Xu and Honglin Jin
}

\begin{abstract}
The current work describes the combined data of three field campaigns, spanning 2009-2013. Their joint gravity and GPS observations thoroughly cover the sites of lithospheric flexure between the Sichuan Basin and the Eastern Tibetan Plateau. The study area's free-air gravity anomalies (FGAs) are updated by using a remove-and-restore algorithm which merges EGM2008 data with in situ observations. These new FGAs show pairs of positive and negative anomalies along the eastern edges of the Tibetan Plateau. The FGAs are used to calculate effective elastic thickness $\left(T_{\mathrm{e}}\right)$ and load ratios ( $F$ ) of the lithosphere. Admittance analysis indicates the $T_{\mathrm{e}}$ of Longmen Shan (LMS) to be $6 \mathrm{~km}$, and profile analysis indicates that the $T_{e}$ of the Sichuan Basin excesses $30 \mathrm{~km}$. The load ratio $\left(F_{1}=1\right)$ confirms that the lithospheric flexure of the LMS area can be attributed solely to the surface load of the crust.
\end{abstract}

Keywords: Free-air gravity anomaly (FGA), Lithospheric flexure, Effective elastic thickness $\left(T_{e}\right)$, Longmen Shan (LMS), EGM2008

\section{Background}

The Tibetan Plateau was formed and continues to be shaped by tectonic forces involved in the collision of the Indian and Eurasian tectonic plates. The area has steep topography at its margins, of which the Longmen Shan (LMS) is one of the steepest. This area has an active tectonic belt, and it has been subjected to two serious earthquakes in the past 8 years $\left(2008 \mathrm{M}_{\mathrm{w}} 7.9\right.$ Wenchuan earthquake and $2013 \mathrm{M}_{\mathrm{w}}$ 6.6 Lushan earthquake). There are four models that researchers use to interpret the abrupt topographic relief across the LMS belt: (1) The channel flow model (Fig. 1a) poses that Tibet's middle and lower crust flow and thrust to the surface in the form of a channel to produce the LMS fault system (Royden et al. 1997; Clark et al. 2005; Klemperer 2006). (2) The crustal shortening model (Fig. 1b) poses that lithospheric-scale escape combines with underthrusting and

*Correspondence: fugy@cea-ies.ac.cn

Key Laboratory of Earthquake Prediction, Institute of Earthquake Science, CEA, Beijing 100036, China

\section{Springer Open}

shortening of the crust beneath the eastern Tibetan Plateau (Tapponnier et al. 2001; Hubbard and Shaw 2009). (3) The wedge model (Fig. 1c) poses that the rigid crust of the Sichuan Basin is wedged into the lower crust of the Songpan-Ganzi block (Cui et al. 1996). (4) The fluid crust model (Fig. 1d) shows the fluid Tibetan crust flowing over a rigid substrate of the Sichuan Basin (Copley and McKenzie 2007).

The lithosphere flexure and load ratio of the LMS area have been studied extensively (Chen et al. 2011; Fielding and McKenzie 2012), and these studies have helped to determine the uplifting mechanism of LMS. However, because the satellite gravity data used in previous studies are of limited accuracy, their results have been contradictory. The study by Chen et al. (2011) supported the channel flow model, but the work of Fielding and McKenzie (2012) gave an opposite result which supported the fluid crust model. These contradictions indicate that higher accurate gravity data should be adopted if the uplifting mechanism of LMS is to be determined. 

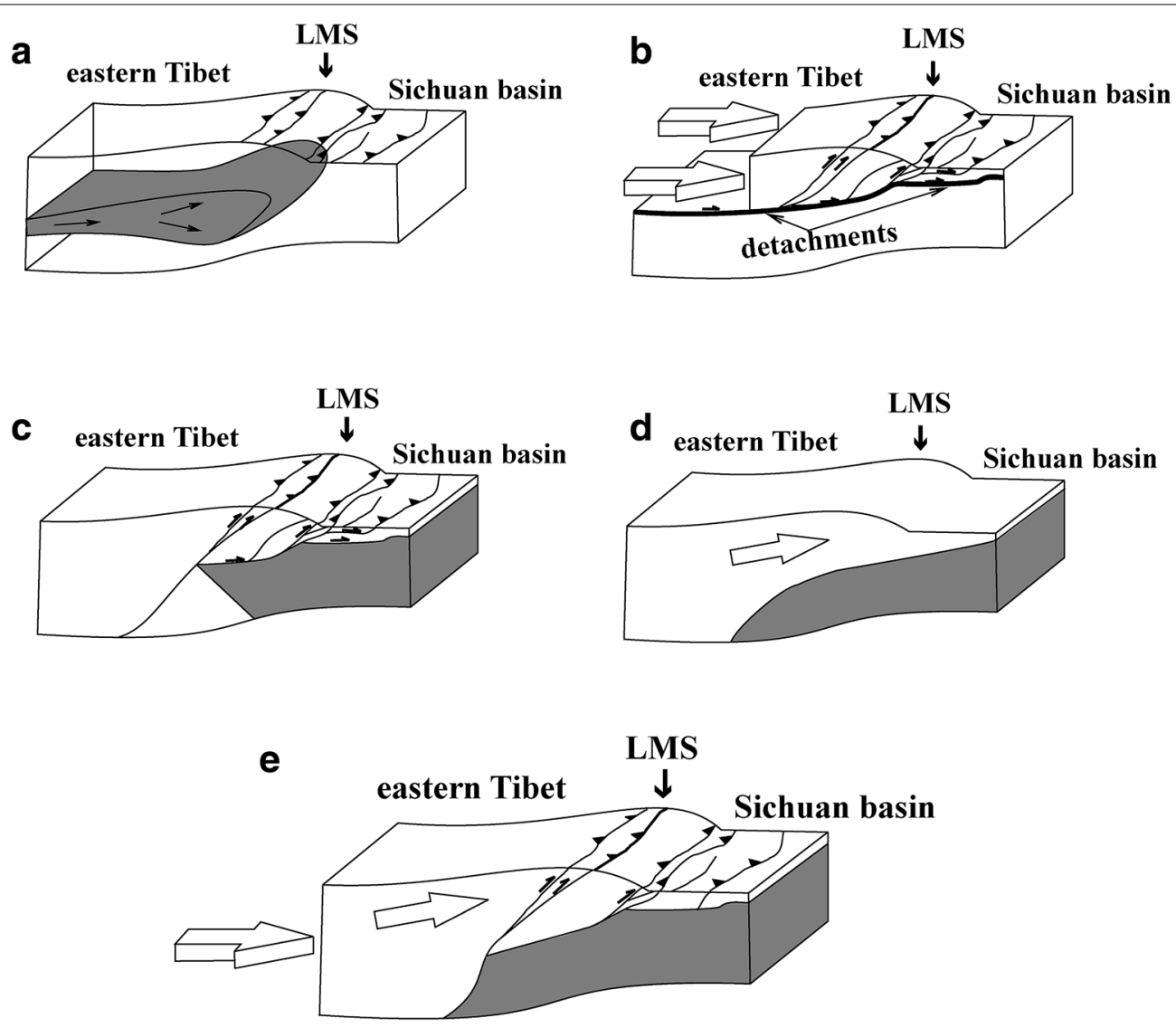

Fig. 1 The sketches of the uplifting mechanisms of the LMS, which proposed by previous studies; a the channel flow model (Royden et al. 1997; Clark et al. 2005; Klemperer 2006); b the crustal shortening model (Tapponnier et al. 2001; Hubbard and Shaw 2009); c the wedge model (Cui et al. 1996); d the fluid crust model (Copley and McKenzie 2007). e The combined model suggested by this study

In this paper, the free-air gravity anomalies (FGAs) around the LMS area are updated using the gravity data from the EGM2008 model (Pavlis et al. 2012) and joint gravity/GPS observations conducted in the LMS area in the past several years. The uplifting mechanism of LMS is investigated by using the load ratios $(F)$ and effective elastic thickness $\left(T_{\mathrm{e}}\right)$ of the study area. The results of this work are more reliable than those of previous works because our results are based on a higher accurate FGAs data.

\section{Gravity anomalies around LMS based on joint gravity/GPS observations}

Figure 2 shows the network of joint gravity and GPS observations in the LMS area. These data came from three sources. First, data were collected from 302 stations at the Sichuan Basin, which were measured in $2012(\mathrm{Fu}$ et al. 2014; Fu and Zhang 2014). These stations make up a reticular structure in the Sichuan Basin. The accuracy of gravity and GPS position (elevation) data are $0.02 \mathrm{mGal}$ and $5 \mathrm{~cm}$, respectively. Second, data were collected from two profiles that cross the northern and central areas of the LMS fault zone. Those data were recorded in 2009, soon after the $\mathrm{Mw} 7.9$ Wenchuan earthquake (Zhang et al. 2014). The accuracy is $0.082 \mathrm{mGal}$ for gravity data, and decimeter level for GPS position (elevation) data. Third, data were collected from two profiles recorded in 2013 at the southern parts of the LMS fault zone after the Mw 6.6 Lushan earthquake (Yang 2014; Yang et al. 2015). The accuracies are $0.02 \mathrm{mGal}$ and decimeter level for gravity and GPS position (elevation) data, respectively. These collected data form a well-shaped network that covers the primary areas of the LMS (Fig. 2), and every campaign includes the absolute gravity station. Therefore, the gravity data are collocated in the uniform system. Here, the gravity anomalies data are derived by the WGS84 reference frame. The estimated FGAs accuracy of three campaigns is less than $0.4 \mathrm{mGal}$ after the gravity reductions (Fu et al. 2014). Here, only the elevation of the GPS position data is used in our study.

The FGAs are derived by using the normal reduction, the height reduction, and the Earth tide reduction. Then, 


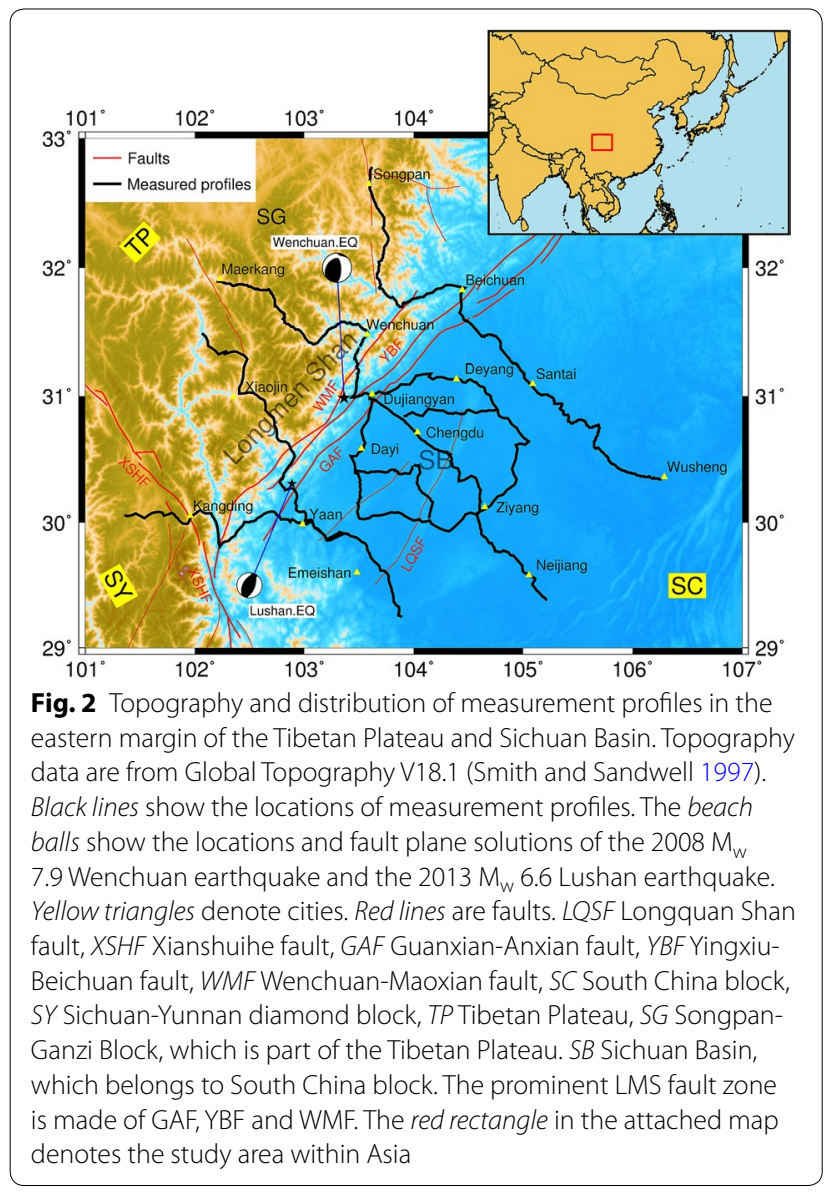

the Bouguer gravity anomalies from these FGAs are determined by using the terrain reduction and the topographic mass reduction (density of $2.67 \mathrm{~g} \mathrm{~cm}^{-3}$ ). Data with a grid spacing of $1^{\prime \prime} \times 1^{\prime \prime}$ from Aster GDEM 2011 are used for the terrain corrections in near field zones $0-2 \mathrm{~km}$. For moderate field zones $2-20 \mathrm{~km}, 5^{\prime \prime} \times 5^{\prime \prime}$ grid spacing is used, and $10^{\prime \prime} \times 10^{\prime \prime}$ is used for far field zones 20-167 km (Fu et al. 2014).

Figure 3 shows the free-air and Bouguer gravity anomalies of the LMS area as indicated by joint gravity and GPS ground observations. A 2D Kriging interpolation is used here to produce a spatial map of these anomalies. As shown in Fig. 3, only the data near the observation stations are presented in color, and the cut-off distance from the stations is $10 \mathrm{~km}$. Data collected far from the observation stations are given in gray contours instead. Overall, the general distribution of the gravity anomalies can be seen by the distribution of the color area in Fig. 3.

More specifically, the FGAs (Fig. 3a) of the study area are negative, with positive anomalies in places. They vary from $-240 \mathrm{mGal}\left(10^{-5} \mathrm{~ms}^{-2}\right)$ to $80 \mathrm{mGal}$. All of the Bouguer gravity anomalies (Fig. $3 \mathrm{~b}$ ) are negative and
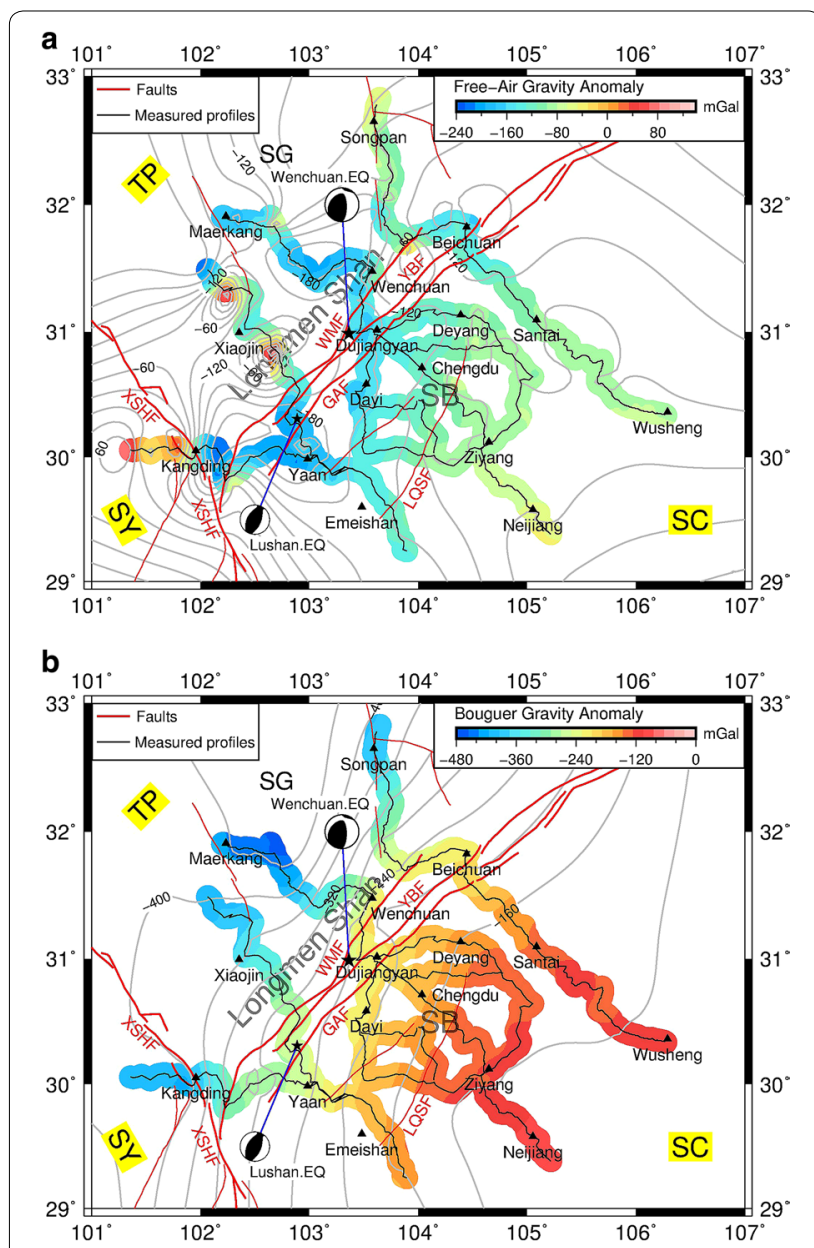

Fig. 3 FGAs (a) and Bouguer gravity anomalies (b) around the LMS area based on the joint gravity and GPS observations. Data along the measurement profiles are show in color, inter- and extrapolated values are shown as contour lines. Cities are indicated using black triangles. LQSF Longquan Shan fault, XSHF Xianshuihe fault, GAF Guanxian-Anxian fault, YBF Yingxiu-Beichuan fault, WMF WenchuanMaoxian fault, SC South China block, SY Sichuan-Yunnan diamond block, TP Tibetan Plateau, SG Songpan-Ganzi Block, which is part of the Tibetan Plateau. SB Sichuan Basin, which belongs to South China block

gradually decreased in magnitude from west to east. The values of Bouguer gravity anomalies in the western part of the study area are found to reach $-480 \mathrm{mGal}$, whereas in the east they are as small as $-80 \mathrm{mGal}$. This is probably related to the overall topography of the Sichuan Basin and eastern Tibetan Plateau, in which the crust thins from west to east. The gray contour lines representing the Bouguer gravity anomalies trend NNE and are parallel to LMS as a whole. However, the greater the distance between the interpolation point and observation station is, the more unreliable the interpolation is. So the least square collocation method (LSC) is adopted in the next section. 


\section{Free-air gravity anomalies around LMS by merging gravity/GPS observations and EGM2008 data}

The GPS and gravity in situ observations of LMS area were made along roads, all of which were located in deep gorges among high mountains. The computed FGAs at the observation stations are undoubtedly of high accuracy. However, the computed FGAs associated with areas far away from observation stations are considered less accurate, because they are profoundly affected by the local topography (Fig. 3a). More specifically, the variation of elevation will induce a change of gravity, and the magnitude is about $0.3086 \mathrm{mGal}$ per meter. Accordingly, attempting to determine FGAs at the other sides of mountains or into deep gorges by conventional interpolation method (Kriging interpolation used in Fig. 3) can involve considerable error.

EGM2008 is a spherical harmonic model of the Earth's gravitational potential, which is formed by merging terrestrial, altimetry-derived, GRACE mission (Tapley et al. 2004), airborne gravity data. Since the EGM2008 was released, it has been continuing to be revised. Up to now, EGM2008 has updated to V23.1 (Sandwell et al. 2014). EIGEN-6C4 (Förste et al. 2014) is also a spherical harmonic model. However, the low-degree components lesser than 235 contain the newest data of GOCE mission (Drinkwater et al. 2003), and the components greater than 235 degrees contain the EGM2008 data. Both of these models are widely used in earth's gravity field studies. Here, we extract the data from EIGEN-6C4 $\left(2190^{\circ}\right)$ and EGM2008 $\left(2190^{\circ}\right)$ by using the locations of the measurement stations described in the previous section and analyze the differences between the two model data and the measurements described above. The results show that the standard deviation of differences between the models and the measurements are $57.81 \mathrm{mGal}$ for EIGEN-6C4 and $47.98 \mathrm{mGal}$ for EGM2008 in this study. On these grounds, the EGM2008 data are used by this study.

During the construction of EGM2008, only low-resolution terrestrial gravity data are available in some areas, which are referred as the proprietary areas (Pavlis et al. 2012). Unfortunately, China is one of these areas, and the high-resolution data of EGM2008 in China are acquired by using LSC to merge the low-resolution gravity data and the gravity data derived from a digital topography model (DTM 2006.0). DTM 2006.0 is a digital topography model including height, depth and thickness information of land, lake, ocean and ice (Pavlis et al. 2012). The differences between terrestrial gravity and EGM2008 data within China have been compared by several studies. The standard deviation of the differences was found to be about $10 \mathrm{mGal}$ in the Sichuan Basin (Fu et al. 2013), and it increased from below $10 \mathrm{mGal}$ in eastern China to more than $50 \mathrm{mGal}$ in the west (Yang et al. 2012). Both the results of the previous studies and the standard deviation between the measurements and EGM2008 data at the end of the previous paragraph indicate considerable errors exist in EGM2008, not only in the short wavelength, but also in the long wavelength over the LMS area.

The EGM2008 data set may have systematic errors in the LMS area. However, it reflects very well the influence of the regional topography. This can make up for the deficiency of the Kriging interpolation method used for Fig. 3, which cannot reflect the influence of the local topography. Merging the modeled EGM2008 data with in situ observations could render the FGAs in the study area more reliable. This may be particularly relevant for locations far from observation stations. Therefore, the remove-and-restore prediction algorithm based on LSC is used to merge in situ measurements with EGM2008 data. This method is frequently used to derive gravity anomaly fields and terrestrial gravity fields from digital topographic models. During the production of EGM2008, the gravity anomalies derived from DTM2006.0 are considered for the reference field (Pavlis et al. 2012). Similarly, the EGM2008 gravitational model serves as the reference field in our study.

The in situ measurements and EGM2008 data are merged as follows. First, FGAs are extracted from EGM2008 at the points where in situ observations are conducted. Then the measurements are removed from the values of the extracted EGM2008 data to acquire the residuals. The residuals between EGM2008 data and measurements are then used to establish an a priori constraint, which represents a linear relationship between the distances between the observation stations and the covariances of the residuals within this distance. Here, the distances between any two stations are divided into discrete intervals $d_{p}$, which are $(2 p-3) \delta<d_{p}<(2 p-1) \delta,(p=2,3,4, \ldots), \quad$ except when $p=1$, the interval is $0<d_{1}<\delta(\delta=5 \mathrm{~km})$, and $\delta$ is the spacing of the $d_{p}$. The covariances are made up of the autocovariances and covariances, which are calculated by the residuals $\Delta g_{i}(1 \leq i \leq n)$ as

$$
\begin{aligned}
& \text { Autocovariance : } C_{\text {res }}(0)=\frac{1}{n} \sum_{i=1}^{n} \Delta g_{i} \Delta g_{i} \\
& \text { Covariance : } C_{\text {res }}\left(d_{p}\right)=\frac{1}{n_{p}} \sum_{i<j}^{n_{p}} \Delta g_{i} \Delta g_{j}
\end{aligned}
$$

where $\mathrm{n}$ is the total number of the measurements, and the $n_{p}$ is the number of measurements whose mutual distance is falling into $d_{p}$. Figure 4 a shows the relationship between the distance and the covariance, and Fig. $4 \mathrm{~b}$ 

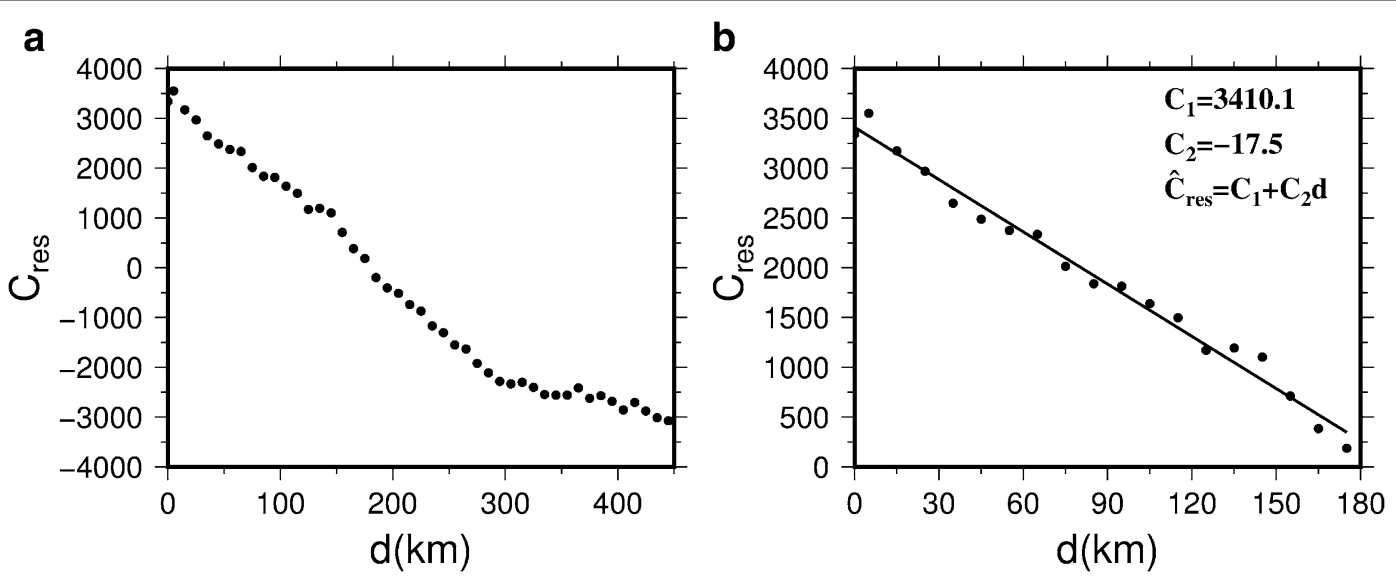

Fig. 4 The covariance values of the FGAs residuals between measurements and EGM2008 data change with the distance $d$ between any two observation stations, and the linear fitting result of the covariance. a The covariance values of the residuals $\left(C_{\text {res }}\right)$ change with the distance between two observation stations. b The linear fitting result of $C_{\text {res }}$ and the covariance function $\hat{C}_{\text {res, }}$ which can be expressed as $\hat{C}_{\text {res }}=C_{1}+C_{2} d . C_{1}$ and $C_{2}$ are the parameters of the covariance function, which are calculated by fitting the $C_{\text {res }}>0$

shows the results of the fit and the covariance function. Here, the residuals $C_{\text {res }}$ less than zero have no meaning, thus the residuals $C_{\text {res }}$ bigger than zero are used to fit the covariance function.

Next, the predicted residual $\Delta \hat{g}_{\text {res }}\left(0.05^{\circ} \times 0.05\right)$ is calculated by using the LSC prediction algorithm (Moritz 1980). The predicted residuals are calculated by

$$
\Delta \hat{g}_{\text {res }}=\hat{C}_{m}\left(C_{m}+D_{\Delta}\right) L
$$

where $\hat{C}_{m}$ is the covariance matrix conducted by the covariance function $\left(\hat{C}_{\text {res }}\right), C_{m}$ is the covariance matrix computed by the residuals, $D_{\Delta}$ is the diagonal matrix of measurement error, and the error is set as $0.4 \mathrm{mGal}$ according to the "Gravity anomalies around LMS based on joint gravity/GPS observations" section. $L$ is the difference between the residuals and its average. Finally, the new FGAs are found around the LMS area (Fig. 5b) by adding the predicted residuals $\Delta \hat{g}_{\text {res }}$ to the corresponding EGM2008 data, and the standard deviation between observed and predicted by LSC is $0.43 \mathrm{mGal}$.

Meanwhile, a new topographic field is established around LMS by merging Global Topography V18.1 (Smith and Sandwell 1997) data with GPS measurements using a similar method. Global Topography V18.1 is a digital elevation model (DEM) data, and it is updated continuously by the recent satellite and geodesy data. Specifically, the terrestrial data of the DEM are based on SRTM30 PLUS with $30^{\prime \prime}$ resolution. Though the accuracy of SRTM is higher than Aster GDEM 2011, not every area is covered well by SRTM data. Therefore, the areas filled by void still persist in the SRTM (Jing et al. 2013). After testing Global Topography V18.1 data with 30", we find no void areas exist in the study area. Considering the updated frequency and accuracy, we chose Global Topography V18.1 as the DEM data in flexure analysis. On the other hand, due to the high resolution $\left(1^{\prime \prime} \times 1^{\prime \prime}\right)$ of Aster GDEM 2011, this DEM data can support more detail near field elevation information than global topography V18.1 around the observation points. Thus, Aster GDEM 2011 is more suitable than SRTM30 PLUS for terrain correction.

A low-pass filter with cut-off at $50 \mathrm{~km}$ wavelength within the frequency domain is used to deal with the FGAs to remove the short-wavelength fluctuation. A Gauss filter with cut-off of $20 \mathrm{~km}$ is used to deal with the elevation data. These different filter processing techniques can extract more relevant information from both the gravity and elevation data, and avoid the disturbance from the high-frequency components simultaneously (Fielding and McKenzie 2012). The filtered FGAs derived from the combination of EGM2008 data and gravity/GPS observations are shown in Fig. 5b.

Pairs of positive and negative anomalies are observed along the edges of the Sichuan Basin and the eastern Tibetan Plateau (Fig. 5a, b). This means that the data collection and processing procedures used here, which are based on the remove-and-restore LSC method, do not alter the total distribution of EGM2008 FGAs in the LMS area. However, the positive anomalies observe in the LMS area shown in the combined gravity field are somewhat smaller than those in EGM2008. Differences like these indicate that the EGM2008 data alone are not inadequate for studying flexure around the LMS area. As described above, the considerable differences between the FGAs in Figs. 3a, 5a and b indicate that merging the model data and in situ observations in the LMS area is 


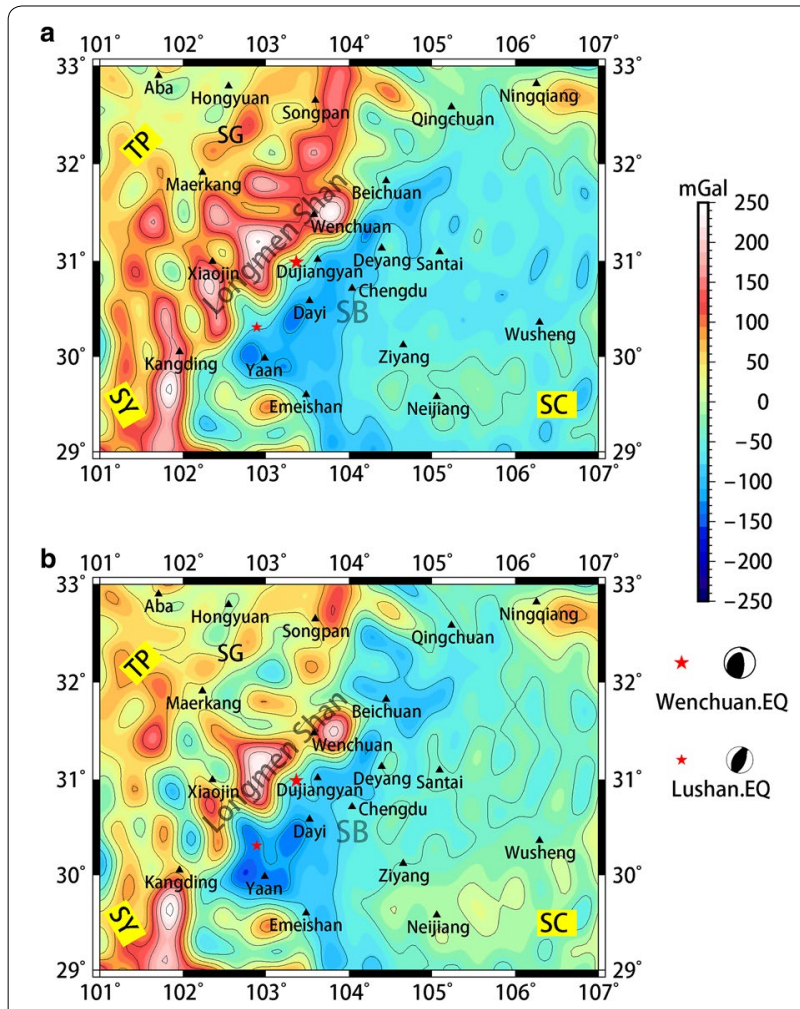

Fig. 5 FGAs around the LMS area. a FGAs of EGM2008. b FGAs derived from the combination of EGM2008 data and in situ observations. Cities are indicated using black triangles. LQSF Longquan Shan fault, XSHF Xianshuihe fault, GAF Guanxian-Anxian fault, YBF YingxiuBeichuan fault, WMF Wenchuan-Maoxian fault, SC South China block, SY Sichuan-Yunnan diamond block, TP Tibetan Plateau, SG SongpanGanzi Block, which is part of the Tibetan Plateau. SB Sichuan Basin, which belongs to South China block

necessary. And more notably, the FGAs in Fig. 3a are the results of the Kriging interpolation, and no filtering is applied. On the contrary, the LSC results in Fig. 5b are treated by a low-pass filter. Therefore, the difference between the FGAs in Figs. 3a and $5 b$ is due to the different data processing method, and the former contains the high-frequency signals and the latter shows the low frequency signals around the study area.

\section{Flexure analysis for the LMS area and Sichuan basin}

The classic isostasy theory assumes that the crust is somewhat malleable and that it floats on the fluid asthenosphere. When the effective thickness of the lithosphere is given, longer wavelengths are more easily compensated than the shorter ones. Thus the regional isostasy is achieved more easily than the local isostasy. Flexural isostasy assumes that loads are supported partially by buoyancy anomalies attributable to deflection of the lithosphere over the fluid asthenosphere and partially by stresses within the elastic lithosphere (Gunn 1943). The flexure of the plate (lithosphere) provides information on the elastic properties of the plate when the load is applied, usually described as the effective elastic thickness $\left(T_{\mathrm{e}}\right)$ of an idealized elastic plate over a fluid asthenosphere (Watts 2001). Generally, greater $T_{\mathrm{e}}$ indicates a stronger lithosphere.

According to the flexure state of the lithosphere of our study area, the spectral method (McKenzie 2003) is here used to estimate the effective elastic thickness $T_{\mathrm{e}}$ and load ratios $F$ of the lithosphere around the LMS area. $T_{\mathrm{e}}$ indicates the strength of the lithosphere, the conversion relation between $T_{\mathrm{e}}$ and flexure rigidity $D$ is expressed as

$$
D=\frac{E T_{\mathrm{e}}^{3}}{12\left(1-\sigma^{2}\right)}
$$

where $E$ denotes the Young's modulus, $\sigma$ denotes the Poisson's ratio. $T_{\mathrm{e}}$ is directly proportional to $D$, when $E$ and $\sigma$ are constant. Then, the bigger $T_{\mathrm{e}}$, the higher the strength of the lithosphere, and vice versa. In other words, the smaller $T_{\mathrm{e}}$, the easier the lithosphere bends.

The method of McKenzie (2003) divides the crust into three layers, one fluid (air), one upper crust, and one lower crust. $F_{\mathrm{i}}$ indicates the ratio of the initial load from different position to the total initial load within the lithosphere. More specifically, if assuming the total initial load is $L_{\text {all }}$, and the initial load from different position is $L_{i}$, then the load ratio $F_{i}=L_{i} / \mathrm{L}_{\text {all }}$. Here, $F_{1}$ represents the load ratio of surface above the upper crust. $F_{2}$ is the load ratio of the interface between the upper and lower crusts, and $F_{3}$ is the load ratio of the interface between the mantle and the crust (Fig. 6). The sum of $F_{1}, F_{2}$, and $F_{3}$ is 1 . Following Fielding and McKenzie (2012), $F_{3}$ is set equal to 0 implying that there is no initial load at the interface between crust and mantle (e.g. magmatic underplating) that was involved in the uplift of the LMS. With $F_{3}=0$, it is then possible to separate the internal load between the upper and lower crusts from the surface load that were present during the uplifting of LMS. Since the sum of $F_{1}$ and $F_{2}$ is 1 , if $F_{2}$ is determined, then $F_{1}$ is also known. The parameters (Fielding and McKenzie 2012) of the crust and mantle are listed in Table 1.

On the one hand, the observed admittance $Z_{c}^{f}$ can be calculated using the measurements of gravity and topography. More specifically, the two-dimensional admittance is calculated by the MultiTaper Method (Thomson 1982; Simons et al. 2000) based on two-dimensional merged gravity and topography data ("Gravity anomalies around LMS based on joint gravity/GPS observations" section), then $Z_{c}^{f}$ (Fig. 7a, black dots) is extracted from the twodimensional admittance by averaging the admittance data of the annular bands with the corresponding wavelength 


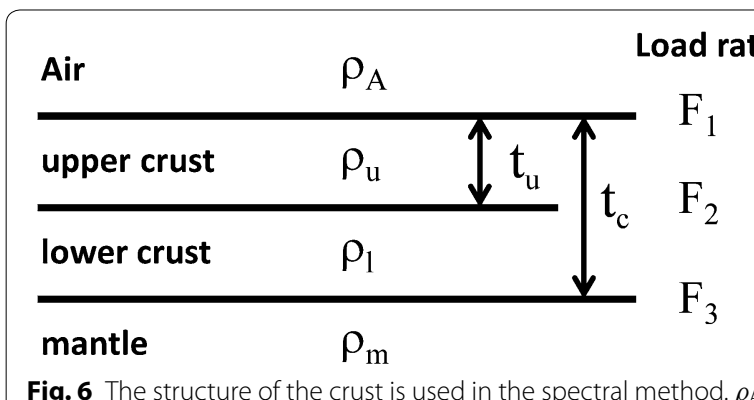

denotes the density of the air, and $\rho_{\mathrm{A}}$ is set to be 0 here; $\rho_{\mathrm{u}}, \rho_{\mathrm{l}}$ and $\rho_{\mathrm{m}}$ denote the densities of the upper crust, lower crust, and mantle, respectively; $t_{u}$ and $t_{c}$ denote the thickness of the upper crust and crust, respectively; $F_{1}, F_{2}$ and $F_{3}$ denote the load ratios from the surface of the crust, the interface between upper crust and lower crust, and the interface between the mantle and the crust, respectively

Table 1 Parameters used in the flexure analysis

\begin{tabular}{lll}
\hline Parameter & Value & Units \\
\hline Upper crust density $\left(\rho_{\mathrm{u}}\right)$ & 2640 & $\mathrm{~kg} / \mathrm{m}^{3}$ \\
Upper crust thickness $\left(t_{\mathrm{u}}\right)$ & 15 & $\mathrm{~km}$ \\
Lower crust density $\left(\rho_{l}\right)$ & 2900 & $\mathrm{~kg} / \mathrm{m}^{3}$ \\
Lower crust thickness $\left(t_{l}\right)$ & 20 & $\mathrm{~km}$ \\
Mantle density $\left(\rho_{\mathrm{m}}\right)$ & 3300 & $\mathrm{~kg} / \mathrm{m}^{3}$ \\
Young modulus $(E)$ & $0.95 \times 10^{11}$ & $\mathrm{~Pa}$ \\
Poisson's ratio $(\sigma)$ & 0.295 & \\
\hline
\end{tabular}

$\rho_{\mathrm{u}}, \rho_{1}$ and $\rho_{\mathrm{m}}$ denote the densities of the upper crust, lower crust, and mantle respectively; $t_{\mathrm{u}}$ and $t_{1}$ denote the thickness of the upper and lower crust, respectively; and $E$ and $\sigma$ denote Young modulus and Poisson's ratio, respectively

as the center, and the standard deviation (Fig. 7a, error bars) is acquired simultaneously (McKenzie 2003). The same method is used to calculate the coherence and phase.

On the other hand, the admittance $Z_{m}^{f}$ can be forwardcalculated based on $T_{\mathrm{e}}$ and $F_{2}$, and every set of $T_{\mathrm{e}}$ and $F_{2}$ corresponds to a model admittance set. Here, the iterated algorithm is used to calculate $Z_{m}^{f}$ with different $T_{\mathrm{e}}$ and $F_{2}$ for fitting the $Z_{\mathcal{C}}^{f}$ where the steps of $T_{\mathrm{e}}$ and $F_{2}$ are $1 \mathrm{~km}$ and 0.01 , respectively. The optimal $T_{\mathrm{e}}$ and $F_{2}$ are finally chosen according to the smallest misfit between $Z_{c}^{f}$ and $Z_{m}^{f}$ Furthermore, the misfit Hf is calculated by

$$
\mathrm{Hf}=\left[\frac{1}{N} \sum_{n=1}^{N}\left(\frac{Z_{c}^{f}-Z_{m}^{f}}{\Delta Z_{c}^{f}}\right)\right]^{1 / 2}
$$

where $N$ is the number of the observed admittance, and $\Delta Z_{c}^{f}$ is the standard deviation of $Z_{c}^{f}$

According to the merged data shown in Fig. 8 (a bigger area than the one shown in Fig. 5b), the $T_{\mathrm{e}}$ in the LMS area is found to be $6 \mathrm{~km}$, and the load ratios are found to be $F_{1}=1$ and $F_{2}=0$ (Fig. $7 \mathrm{a}, \mathrm{d}$ ). These load ratios indicate that the initial load in the LMS area is at the surface of the crust. The coherence between FGAs and topography is high across all wavelengths (Fig. 7c), and the phase of complex admittance (Fig. 7b) is near zero. These findings are consistent with the results of the load ratios, also support that the initial load is at the surface of the crust.

As described above, the $T_{\mathrm{e}}$ is acquired by the spectral method, which is the flexure analysis method of the frequency domain. This result is determined by the principal component of the frequency domain, which in the spatial domain, corresponds to areas with greatest changes (in topography or gravity). In our study area, the topography and FGAs are found to vary steeply across the eastern edges of the Tibetan Plateau, which response to the principal component. However, there were relatively mild variations over the Sichuan Basin. Therefore, considering the difference between the Sichuan Basin and eastern Tibetan Plateau, the estimated $T_{\mathrm{e}}=6 \mathrm{~km}$ is considered suitable for the eastern Tibetan Plateau which includes the LMS area. This result indicates that the strength of the crust of the eastern Tibetan Plateau is low, and it is easy to flexure. Because of the mild variations of the topography, the $T_{\mathrm{e}}$ of the Sichuan Basin is not determined by the spectral method but requires a different approach.

With the decrease of regional scale, the accuracy of estimation by spectral methods will decline (PérezGussinyé et al. 2004). Considering the wave number $k=\sqrt{k x^{2}+k y^{2}}(k x$ and $k y$ are the wave number of each side of the square study area) and the wavelength $\lambda=2 \pi / k$, if we assume that the scale of the Sichuan Basin is $350 \times 350 \mathrm{~km}$, then the maximum wavelength of admittance is nearly $250 \mathrm{~km}$. However, this maximum value is too short to support sufficient information to constrain the admittance in the long wavelength (Fig. 7a). According to this principle, using spectral method to estimate the $T_{\mathrm{e}}$ of narrow Sichuan Basin is here inadequate, so a profile method is used to estimate the $T_{\mathrm{e}}$ in Sichuan Basin.

NW-SE-oriented profiles of FGAs that stacked from the Sichuan Basin are used here, and they are shown in yellow rectangle in Fig. 8. The stacking method described by McKenzie (2003) is used here. Specifically, the individual profiles are first stacked in the rectangular area to produce a mean profile. Then, a scaling factor $S_{i}$ is used to multiply each separate profile, which is determined by

$$
S_{i}=\left[\sum_{j=1}^{N}\left(g_{i j}-\bar{g}_{i}\right)\left(\bar{g}_{j}-\bar{g}\right)\right] / \sum_{j=1}^{N}\left(g_{i j}-\bar{g}_{i}\right)^{2}
$$

where $g_{\mathrm{ij}}$ is the FGA of $\mathrm{jth}$ point of ith profile, $\bar{g}_{i}$ is the average of the FGAs of ith profile, $\bar{g}_{j}$ (Fig. 9a, black 

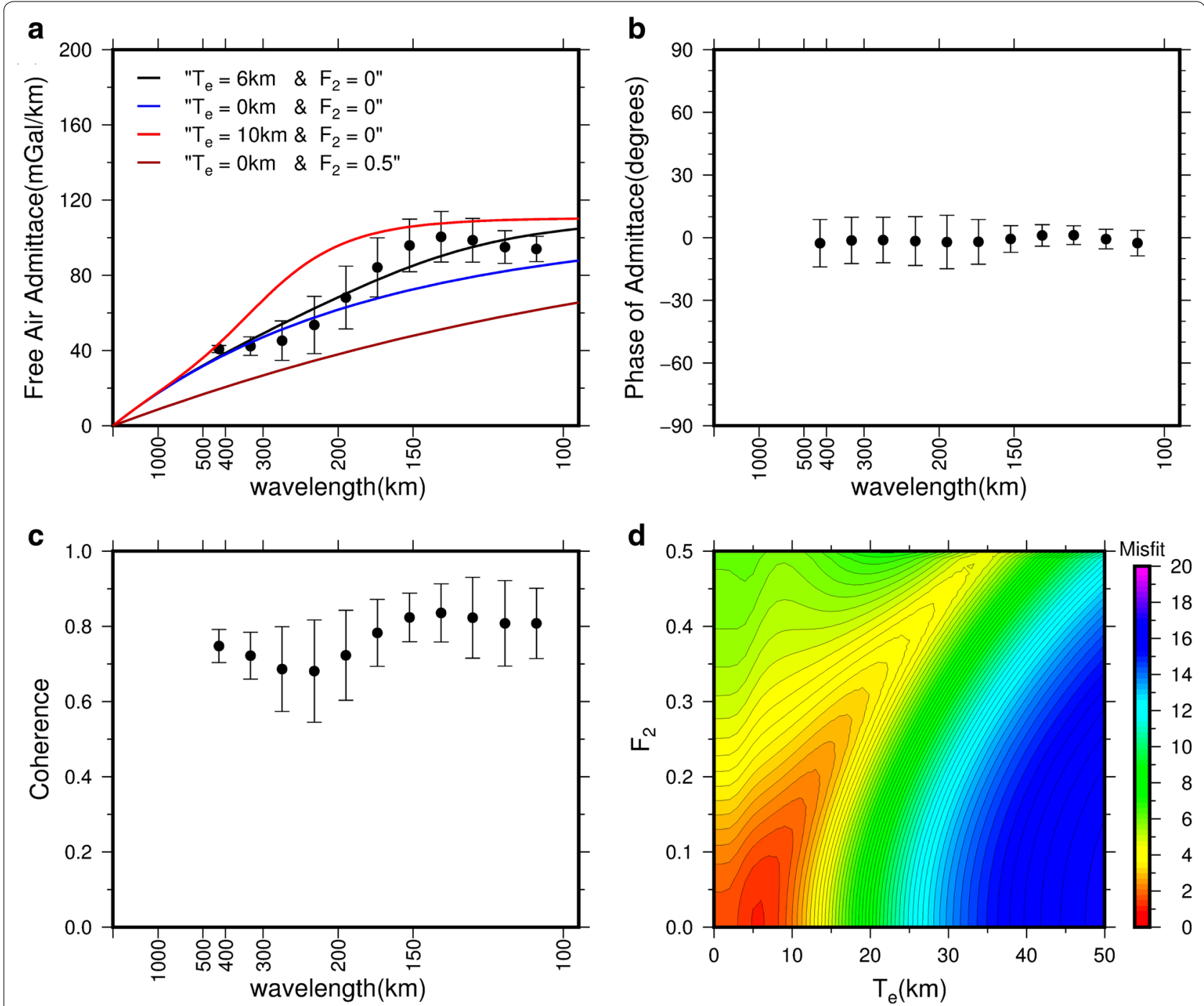

Fig. 7 Flexure analysis from two-dimensional admittance between FGAs and topography around LMS area. Error bars are $1 \sigma$ (standard deviation). a Admittance as a function of wavelength from observed data and forward calculations. $F_{3}$ is set to zero. $\mathbf{b}$ Complex phase of admittance between FGAs and topography. c Coherence magnitude between FGAs and topography. $\mathbf{d}$ Misfit between the admittance deduced from the FGAs around LMS and the model admittance which is a function of $T_{e}$ and $F_{2}$. The optimal fit is at $T_{e}=6 \mathrm{~km}$ and $F_{2}=0$

curve) is the FGAs of the mean profile, $\bar{g}$ is the average of the mean profile, and $N$ is the number of the FGAs of each profile. Here, $N$ is equal to 30 , and $i(1 \leq i \leq 10)$ denotes the profile number. The scaling factor is used to minimize the difference between each individual profile and the mean. In this way, the standard deviation $\sigma_{j}$ (Fig. 9a, dash line) of the profiles could be reduced without changing their shapes, and $\sigma_{j}$ can be expressed as

$$
\sigma_{j}=\sqrt{\frac{1}{M} \sum_{i=1}^{M}\left(g_{i j} S_{i}-\frac{1}{M} \sum_{i=1}^{M} g_{i j} S_{i}\right)^{2}}
$$

$M$ is the total number of the individual profiles, and $M$ is equal to 10.

The flexure model used here is based on the bending plate model (Turcotte and Schubert 2014). This method assumes that the crust is a continuous elastic plate floating on the mantle and that there exists a bending moment at the end of the plate. The FGAs $g_{j}^{c}$ (Fig. 9a, gray dots) of the flexure model is calculated by

$$
\begin{aligned}
g_{j}^{c}= & \exp \left(-x_{j} / \alpha\right)\left[C_{1} \cos \left(x_{j} / \alpha\right)+C_{2} \sin \left(x_{j} / \alpha\right)\right] \\
& +C_{3}+C_{4} x_{j}
\end{aligned}
$$




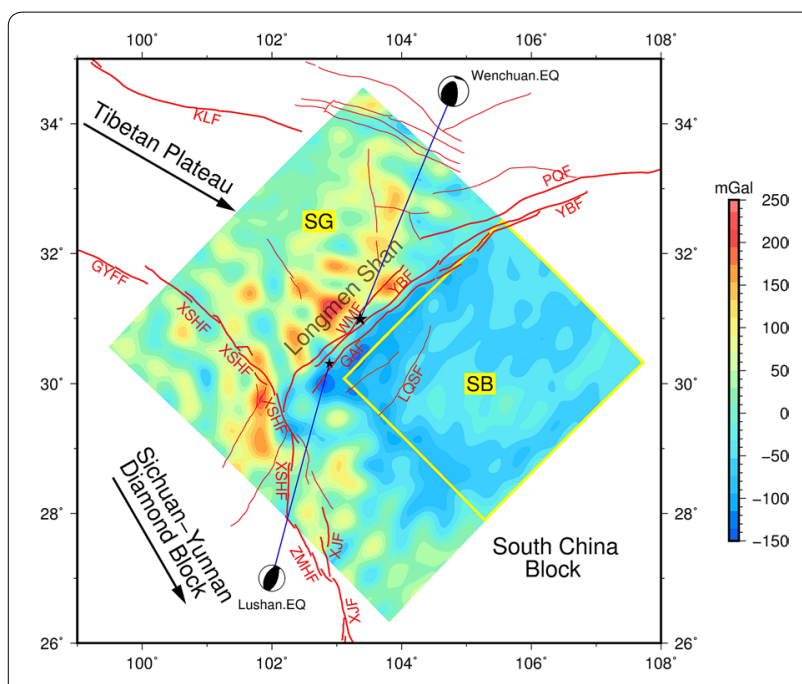

Fig. 8 FGAs that are used to analyze the flexure in LMS area and Sichuan Basin. Arrows denote the movement direction of blocks. The data of yellow rectangle are used in the profile method to calculate the $T_{e}$ of the Sichuan Basin. LQSF Longquan Shan fault, XSHF Xianshuihe fault, GAF Guanxian-Anxian fault, YBF Yingxiu-Beichuan fault, WMF Wenchuan-Maoxian fault, SC South China block, SY SichuanYunnan diamond block, TPTibetan Plateau, SG Songpan-Ganzi Block, which is part of the Tibetan Plateau. SB Sichuan Basin, which belongs to South China block

$$
\alpha=\left[\mathrm{ET}_{\mathrm{e}}^{3} / 3 g\left(\rho_{\mathrm{m}}-\rho_{\mathrm{s}}\right)\left(1-\sigma^{2}\right)\right]^{1 / 4}
$$

where $x_{j}$ is the distance along the profile, $g=9.81 \mathrm{~m} / \mathrm{s}^{2}$, $\rho_{s}$ is the density of the sedimentary layer, and this value is equal to $2400 \mathrm{~kg} / \mathrm{m}^{3}$ (Fielding and McKenzie, 2012). The values of $E, \sigma$ and $\rho_{m}$ are given in Table 1 , and $C_{1}, C_{2}$, $C_{3}$ and $C_{4}$ are constants, calculated by minimizing $H$ for each value of $T_{\mathrm{e}} . H$ is the misfit between $g_{\mathrm{j}}^{\mathrm{c}}$ and $\bar{g}_{j}$, and it can be expressed as:

$$
H\left(T_{e}\right)=\left[\frac{1}{N} \sum_{j=1}^{N}\left(\frac{g_{j}^{c}-\bar{g}_{j}}{\sigma_{j}}\right)^{2}\right]^{1 / 2}
$$

This is similar with the admittance method, when $H$ achieves the smallest value, the optimal $T_{\mathrm{e}}$ is determined.

According to the profile method described above, the best fitting result involved a $T_{\mathrm{e}}$ of Sichuan Basin is $59.3 \mathrm{~km}$, but any $T_{\mathrm{e}}>30 \mathrm{~km}$ fits the stacked profile nearly as well (Fig. 9b). This result indicates that the strength of the Sichuan Basin is high, and comparing to the low strength of the crust of the eastern Tibet Plateau, it is hard to flex.

\section{Discussions}

Studies of $T_{\mathrm{e}}$ in the eastern Tibetan Plateau and the Sichuan Basin are here reviewed. Braitenberg et al. (2003) estimated Tibetan's $T_{\mathrm{e}}$ to be between 10 and $30 \mathrm{~km}$ with the smallest values in eastern Tibet. Jiang and Jin (2005) showed the range of $T_{\mathrm{e}}$ in eastern Tibetan to be $36-38 \mathrm{~km}$. Jordan and Watts (2005) found the $T_{\mathrm{e}}$ of the Tibetan Plateau to range from 5 to $35 \mathrm{~km}$, and they recorded the smallest values in southeastern Tibetan. Mao et al. (2012) estimated $T_{\mathrm{e}}$ in the same area as this study (Fig. 2) to be $10-40 \mathrm{~km}$ and the one in Sichuan Basin over $30 \mathrm{~km}$. Fielding and McKenzie (2012) estimated the $T_{\mathrm{e}}$ in eastern Tibetan Plateau to be $7 \mathrm{~km}$ and the $T_{\mathrm{e}}$ in the Sichuan Basin to exceed $10 \mathrm{~km}$. Chen et al. (2014) estimated the $T_{\mathrm{e}}$ within eastern margin of Tibetan Plateau to be within a range of $10-20 \mathrm{~km}$ and estimated that the $T_{\mathrm{e}}$ in the Sichuan Basin would range from 30 to $40 \mathrm{~km}$. The previous studies listed above used low-resolution grid data (Braitenberg
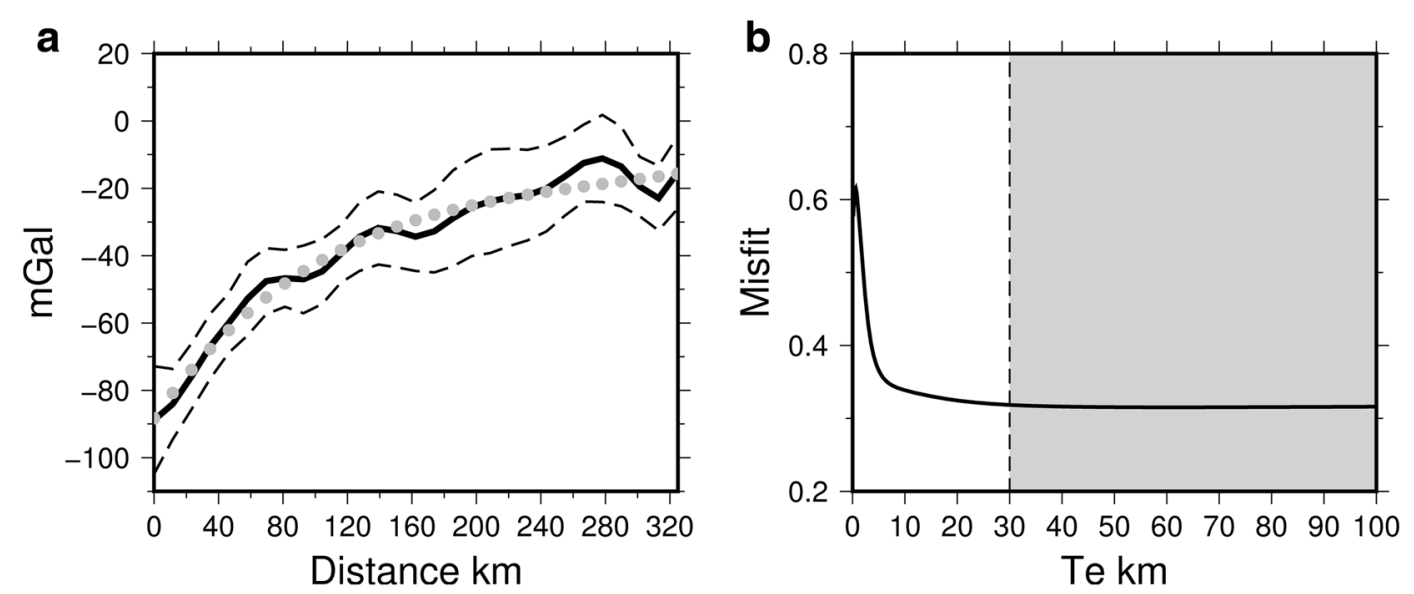

Fig. 9 Stacked profiles of Sichuan Basin and flexure fitting results. a Mean stacked profile (solid line) deduced from the FGAs over Sichuan Basin, standard deviation range (dashed lines), and best fit flexure model (dotted line) with $T_{e}=59.3 \mathrm{~km}$ (optimal result). Distance is the long edge of the yellow rectangle in Fig. 8. b Misfit between the flexure deduced from the FGAs over Sichuan Basin and the flexure model which is a function of $T_{e}$ the gray area shows the region of best fit 
et al. 2003; Jiang and Jin 2005; Jordan and Watts 2005) or model data (Mao et al. 2012; Fielding and McKenzie 2012; Chen et al. 2014) only, and the method is based on profile method (Braitenberg et al. 2003; Jiang and Jin, 2005; Jordan and Watts 2005) or admittance method (Mao et al. 2012; Fielding and McKenzie 2012; Chen et al. 2014). The different data and different methods make the results from previous studies above different. However, their results somewhat indicate that the crust of the eastern Tibet Plateau is of lower strength than the crust of the Sichuan Basin, and this is consistent with the results of this paper.

Here, the data used in this study merge the ground measurements and EGM2008 data. The measurements with high accuracy and good distribution around the LMS area are not attended in the previous studies. The results of the spectral and profile method analysis are combined here, and the $T_{\mathrm{e}}$ in the eastern Tibetan Plateau is estimated to be $6 \mathrm{~km}$, including the LMS area. The $T_{\mathrm{e}}$ in the Sichuan Basin is found to be greater than $30 \mathrm{~km}$. The results gathered here are relatively close to those reported by Fielding and McKenzie (2012). The differences between the results of the present work and those of Fielding and McKenzie (2012) are attributed to the usage of in situ GPS elevation and gravity measurements.

On the one hand, $T_{\mathrm{e}}=6 \mathrm{~km}$ in the eastern Tibetan Plateau and $T_{\mathrm{e}}>30 \mathrm{~km}$ in Sichuan Basin indicate that the curst with low strength in eastern Tibet Plateau is easier to deform than the crust of the Sichuan Basin. This corresponds to the fluid crust model (Copley and McKenzie 2007), which the eastern Tibetan Plateau crust (with a low viscosity $10^{20} \mathrm{~Pa} \mathrm{~s}$ ) flowing over the strong Sichuan Basin lithosphere (with a high viscosity $10^{22} \mathrm{~Pa}$ ). On the other hand, the load ratios of the current calculation are $F_{1}=1$ and $F_{2}=0$. Here, $F_{1}=1$ indicates that the initial load of LMS area is located at the crust's surface and this result supports the crustal shortening model (Hubbard and Shaw 2009) and the fluid crust model (Copley and McKenzie 2007) for the uplifting mechanism of the LMS. $F_{2}$ refers to the contribution between upper crust and lower crust, which corresponds to the location of the channel flow and tectonic wedge of the middle and lower crusts in Tibetan Plateau. Thus, $F_{2}=0$ indicates that the channel flow and tectonic wedge make absolutely no contribution to the uplift of LMS.

As describes above, the results of the current calculation support the fluid crust model and the crustal shortening model. Therefore, we give a model (Fig. 1e) combining both models. The collision between the soft crust of the eastern Tibetan Plateau and the hard crust of the Sichuan Basin happened in the eastward movement of the Tibetan Plateau. Then, the eastern Tibet Plateau crust was deforming, breaking and thrusting over the Sichuan Basin crust. Simultaneously, the LMS area, which is the collision zone, uplifted. This tectonic model agrees with the hypothesis published by $\mathrm{Fu}$ et al. (2014), who said that the Longquan Shan fault zone is a high-angle, deeply rooted fault zone broken by the load of LMS. Meanwhile, the result of the deep seismic reflection profile, which combined with geological, GPS, and geochemical evidence, strongly suggests that the Sichuan basin crust extends beneath the eastern Tibet Plateau (Guo et al. 2013). This is consistent with our model.

\section{Conclusions}

Based on the in situ joint gravity and GPS observations conducted in the past several years, a dense network was constructed between the Sichuan Basin and the eastern Tibetan Plateau (Fig. 2). Then, the regional FGAs and Bouguer gravity anomalies of the study area are updated. The accuracy of the computed FGAs at and near the observation stations is high. Yet the one associated with areas far away from the observation stations is not high, because FGAs can be profoundly affected by the local topography. To render FGAs for areas far from observation stations more accurate (Fig. 2), the in situ observations are merged with EGM2008 data using a remove-and-restore algorithm (Pavlis et al. 2012). The new FGAs fields show pairs of positive and negative anomalies along the edges of the Sichuan Basin and the eastern Tibetan Plateau (Fig. 5b), in much more detail than the results found using EGM2008 data alone.

The new FGAs are used to calculate $T_{\mathrm{e}}$ and $F$ in the LMS area through two-dimensional admittance analysis (McKenzie 2003). The results show that the $T_{\mathrm{e}}$ in the LMS area is $6 \mathrm{~km}$. Profile analysis indicate the $T_{\mathrm{e}}$ in Sichuan Basin exceed $30 \mathrm{~km}$. Small $T_{\mathrm{e}}$ in the LMS area and great $T_{\mathrm{e}}$ in Sichuan Basin support the fluid crust model of the eastern Tibetan Plateau crust flowing over strong Sichuan Basin lithosphere (Copley and McKenzie 2007). $F_{1}$ is the load ratio indicated the initial load from the surface of the crust, and $F_{2}$ is the load ratio indicated the initial load from the interface between the lower and upper crust. $F_{1}=1$ and $F_{2}=0$ indicate that the lithospheric flexure of the LMS area is entirely attributed to the surface load. This is consistent with the crustal shortening model and the fluid crust model of the uplifting of the LMS. This study recommends a uplifting mechanism of the LMS, which combines the crustal shortening model and the fluid crust model. The eastern Tibetan crust with a low strength was not only thrusting over the Sichuan basin crust with a high strength, but also breaking and shortening simultaneously. With this processing, the LMS was uplifting and forming one of the steepest topographic marginal areas around the Tibetan Plateau.

\section{Authors' contributions}

YS and GF have made substantial contributions to conception and design, acquisition of data, analysis and interpretation of data, and ZW, TL, CX, and HJ 
carried out the observations of the relative gravity data in Longmen Shan area. All authors read and approved the final manuscript.

\section{Acknowledgements}

The authors are grateful to Dr. Sofie Gradmann and the other anonymous reviewers for their helpful comments. This study is financially supported by National Science Foundation of China $(41574071,41331066,41461164004)$ and Basic Research Project of Institute of Earthquake Science, Chinese Earthquake Administration (IES20150201). Data supporting this article are available in within this article.

\section{Competing interests}

The authors declare that they have no competing interests.

Received: 7 April 2016 Accepted: 22 September 2016

Published online: 06 October 2016

\section{References}

Braitenberg C, Wang Y, Fang J et al (2003) Spatial variations of flexure parameters over the Tibet-Quinghai plateau. Earth Planet Sci Lett 205(3):211-224

Chen S, Wang Q, Zhu Q et al (2011) Temporal and spatial features of isostasy anomaly using gravitational admittance model at eastern margin of Tibetan Plateau. Chin J Geophys 54(1):22-34

Chen B, Liu J, Kaban MK et al (2014) Elastic thickness, mechanical anisotropy and deformation of the southeastern Tibetan Plateau. Tectonophysics 637:45-56

Clark MK, Bush JW, Royden LH (2005) Dynamic topography produced by lower crustal flow against rheological strength heterogeneities bordering the Tibetan Plateau. Geophys J Int 162(2):575-590

Copley A, McKenzie D (2007) Models of crustal flow in the India-Asia collision zone. Geophys J Int 169(2):683-698

Cui ZZ, Chen JP, Wu L (1996) Deep crustal structure and tectonics in Huashixia-Shaoyang profile. Geol. Publ, Beijing, pp 156-168

Drinkwater MR, Floberghagen R, Haagmans R et al (2003) GOCE: ESA's first earth explorer core mission Earth gravity field from space-from sensors to earth sciences. Springer, Newyork city, pp 419-432

Fielding EJ, McKenzie D (2012) Lithospheric flexure in the Sichuan Basin and Longmen Shan at the eastern edge of Tibet. Geophys Res Lett 39:L09311. doi:10.1029/2012GL051680

Förste C, Bruinsma SL, Abrikosov O et al (2014) EIGEN-6C4 The latest combined global gravity field model including GOCE data up to degree and order 2190 of GFZ potsdam and GRGS toulouse. GFZ Data Services. doi:10.5880/icgem

Fu G, Zhang G (2014) Significant isostatic imbalance near the seismic gap between the M8.0 Wenchuan and the M7.0 Lushan earthquakes. Chin Sci Bull 59(34):4774-4780

Fu G, Zhu Y, Gao S et al (2013) Discrepancies between free air gravity anomalies from EGM2008 and the ones from dense gravity/GPS observations at west Sichuan Basin. Chin J Geophys 56(11):376-3769

Fu G, Gao S, Freymueller JT et al (2014) Bouguer gravity anomaly and isostasy at western Sichuan Basin revealed by new gravity surveys. J Geophys Res Solid Earth 119(4):3925-3938

Gunn R (1943) A quantitative evaluation of the influence of the lithosphere on the anomalies of gravity. J Franklin Inst 236:47-65

Guo X, Gao R, Keller GR et al (2013) Imaging the crustal structure beneath the eastern Tibetan Plateau and implications for the uplift of the Longmen Shan range. Earth Planet Sci Lett 379:72-80

Hubbard J, Shaw JH (2009) Uplift of the Longmen Shan and Tibetan plateau, and the 2008 Wenchuan $(M=7.9)$ earthquake. Nature 458(7235):194-197

Jiang $X$, Jin Y (2005) Mapping the deep lithospheric structure beneath the eastern margin of the Tibetan Plateau from gravity anomalies. J Geophys Res Solid Earth. doi:10.1029/2004JB003394

Jing C, Shortridge A, Wu SLJ (2013) Comparison and validation of SRTM and ASTER GDEM for a subtropical landscape in southeastern China. Int J Digit Earth 7(12):969-992
Jordan TA, Watts AB (2005) Gravity anomalies, flexure and the elastic thickness structure of the India-Eurasia collisional system. Earth Planet Sci Lett 236(3):732-750

Klemperer SL (2006) Crustal flow in Tibet: a review of geophysical evidence for the physical state of Tibetan lithosphere, in Channel Flow. Ductile extrusion and exhumation of lower mid-crust in continental collision zones. Geol Soc London 268:39-70

Mao X, Wang Q, Liu S et al (2012) Effective elastic thickness and mechanical anisotropy of South China and surrounding regions. Tectonophysics 550:47-56

McKenzie D (2003) Estimating $T_{e}$ in the presence of internal loads. J Geophys Res Solid Earth. doi:10.1029/2002JB001766

Moritz H (1980) Advanced physical geodesy. Abacus Press, Preston

Pavlis NK, Holmes SA, Kenyon SC et al (2012) The development and evaluation of the earth gravitational model 2008 (EGM2008). J Geophys Res Solid Earth 117:B04406. doi:10.1029/2011JB008916

Pérez-Gussinyé M, Lowry AR, Watts AB et al (2004) On the recovery of effective elastic thickness using spectral methods: examples from synthetic data and from the Fennoscandian Shield. J Geophys Res 109(109):235-243

Royden LH, Burchfiel BC, King RW et al (1997) Surface deformation and lower crustal flow in eastern Tibet. Science 276(5313):788-790

Sandwell DT, Müller RD, Smith WH et al (2014) New global marine gravity model from CryoSat-2 and Jason-1 reveals buried tectonic structure. Science 346(6205):65-67

Simons FJ, Zuber MT, Korenaga J (2000) Isostatic response of the Australian lithosphere: estimation of effective elastic thickness and anisotropy using multitaper spectral analysis. J Geophys Res Solid Earth 105(B8):19163-19184

Smith WH, Sandwell DT (1997) Global sea floor topography from satellite altimetry and ship depth soundings. Science 277(5334):1956-1962

Tapley BD, Bettadpur S, Watkins M et al (2004) The gravity recovery and climate experiment: mission overview and early results. Geophys Res Lett 31(9):1-4

Tapponnier P, Xu Z, Roger F et al (2001) Oblique stepwise rise and growth of the Tibet Plateau. Science 294:1671-1677

Thomson DJ (1982) Spectrum estimation and harmonic analysis. Proc IEEE 70(9):1055-1096

Turcotte DL, Schubert G (2014) Geodynamics. Cambridge University Press, Cambridge

Watts AB (2001) Isostasy and flexure of the lithosphere. Cambridge University Press, Cambridge

Yang G (2014) Study on the crustal structure and dynamic environment in the eastern boundary region of Sichuan-Yunnan Block, Postdoctoral Thesis, University of Chinese Academy of Sciences

Yang J, Zhang X, Zhang F et al (2012) On the accuracy of EGM2008 earth gravitational model in Chinese Mainland. Progress in Geophysics 4:1298-1306. doi:10.6038/j.issn. 1004-2903.2012.04.003

Yang G, Shen C, Wu G et al (2015) Bouguer gravity anomaly and crustal density structure in Jinchuan-Lushan-Qianwei profile. Chin J Geophys 58(7):2424-2435

Zhang Y, Teng J, Wang Q et al (2014) Density structure and isostatic state of the crust in the Longmenshan and adjacent areas. Tectonophysics 619:51-57

\section{Submit your manuscript to a SpringerOpen ${ }^{\odot}$ journal and benefit from:}

- Convenient online submission

- Rigorous peer review

- Immediate publication on acceptance

- Open access: articles freely available online

- High visibility within the field

- Retaining the copyright to your article

Submit your next manuscript at $\mathbf{s p r i n g e r o p e n . c o m ~}$ 